\title{
AIRFLOW MEASUREMENT AT FORMULA STUDENT CAR
}

\author{
Šebela K.*, Janoušek M.**
}

\begin{abstract}
Cooling modern car is difficult topic influencing external and internal aerodynamics. Air used to cool down engine and accessories influence car drag and increase fuel consumption. Evaluating design of car is done in wind tunnel with steady conditions. Air flowing thru heat exchanger must be measured to compare models and simulation. Method for measuring air mass flow is demonstrated at Formula Student car equipped with measuring propeller located downstream of cooling fan. Pressures are measured for control and provide data which are necessary to evaluate cooling design. Recirculation is addressed and method how to deal with this effect is presented. By using air guide upstream heat exchanger air mass flow is increased at high speed and at low speed recirculation is eliminated.
\end{abstract}

Keywords: Airflow measurement, Cooling, Wind tunnel, Formula Student, Air recirculation.

\section{Introduction}

To provide optimal operating condition for internal combustion engine cooling of head, cylinder, piston, and other parts is needed. Low operating temperature of surfaces leads to power loss and increase fuel consumption (Shayler et al., 2005). High temperature causes problems with higher engine thermal stress and problem with knock (Singh et al., 2017).

Heat is exchanged in compact heat exchanger (HX) where coolant is forced by pump and air by combination of ram pressure and fan. Coolant flow is easily measurable by flow meter positioned at inlet or outlet pipe from heat exchanger. Air is however much harder to measure and distribution of air is not close to ideal as at coolant side. Maldistribution of air leads to underperformance of heat exchanger and increase pressure loss thru heat exchanger (Aslam et al., 2012).

Cooling accounts for $5 \div 10 \%$ of total aerodynamics drag at road cars (Baskar et al., 2015). Drag causes increase in aerodynamics resistance and increase fuel consumption. At racing cars cooling drag increase lap time and disturb flow around car. There is important to distinguish between drag caused by inlet, HX and outlet of the air from the air path (Zhang et al., 2018). For assess of design aero parts and HX measurements are important to validate models and simulation to understand current situation and make changes at whole packet. Affected air should be exploit at cooling and therefore increased efficiency should be targeted.

Recirculation in a bad way influence cooling at low speed and decreases cooling capacity by significant quantity. Recirculation influence air, which flows from downstream of HX to front side and increase inlet temperature of air. This can occur at low speed with poor design and by using powerful fan. Pressure behind HX can be higher than at the front and air do not leave engine bay or sidepod and recirculate around HX. Counter measures eliminating recirculation include adjustment of sealing around HX and exit area for air and using control strategy for fan to change speed during operation.

\footnotetext{
* Ing. Kamil Šebela: Institute of Automotive Engineering, Faculty of Mechanical Engineering, Brno University of Technology, Technická 2896, 616 00, Brno, CZ, 170365@vutbr.cz

** Ing. Michal Janoušek, PhD.: Institute of Automotive Engineering, Faculty of Mechanical Engineering, Brno University of Technology, Technická 2896, 616 00, Brno, CZ, Michal.Janousek@vutbr.cz
} 


\section{Methods}

\subsection{Description}

Measuring is performed at Formula Student car Dragon 8 from team TU Brno Racing. Car is powered by $450 \mathrm{~cm}^{3}$ single cylinder turbocharged engine producing $63 \mathrm{~kW}$ at $10500 \mathrm{~min}^{-1}$. Engine is cooled by plain water and coolant is pumped by mechanical centrifugal pump. Coolant HX is located at right side inside sidepod. Car runs at Formula Student tracks with average speed at $60 \div 70 \mathrm{~km} / \mathrm{h}$ with load changes for $22 \mathrm{~km}$ mostly in summer condition.

For asses of air flow and whole design measurement method is developed to understand changes at cooling package and evaluate its performance. Measurement is done in wind tunnel provided by Jaroš company. There is nozzle with dimension of 2.5 and $1.5 \mathrm{~m}$ with capability of speed up to $160 \mathrm{~km} / \mathrm{h}$. Car is placed at standing floor with fixed wheels. Wind speed is altered from 0 to $100 \mathrm{~km} / \mathrm{h}$ in $10 \mathrm{~km} / \mathrm{h}$ increments.

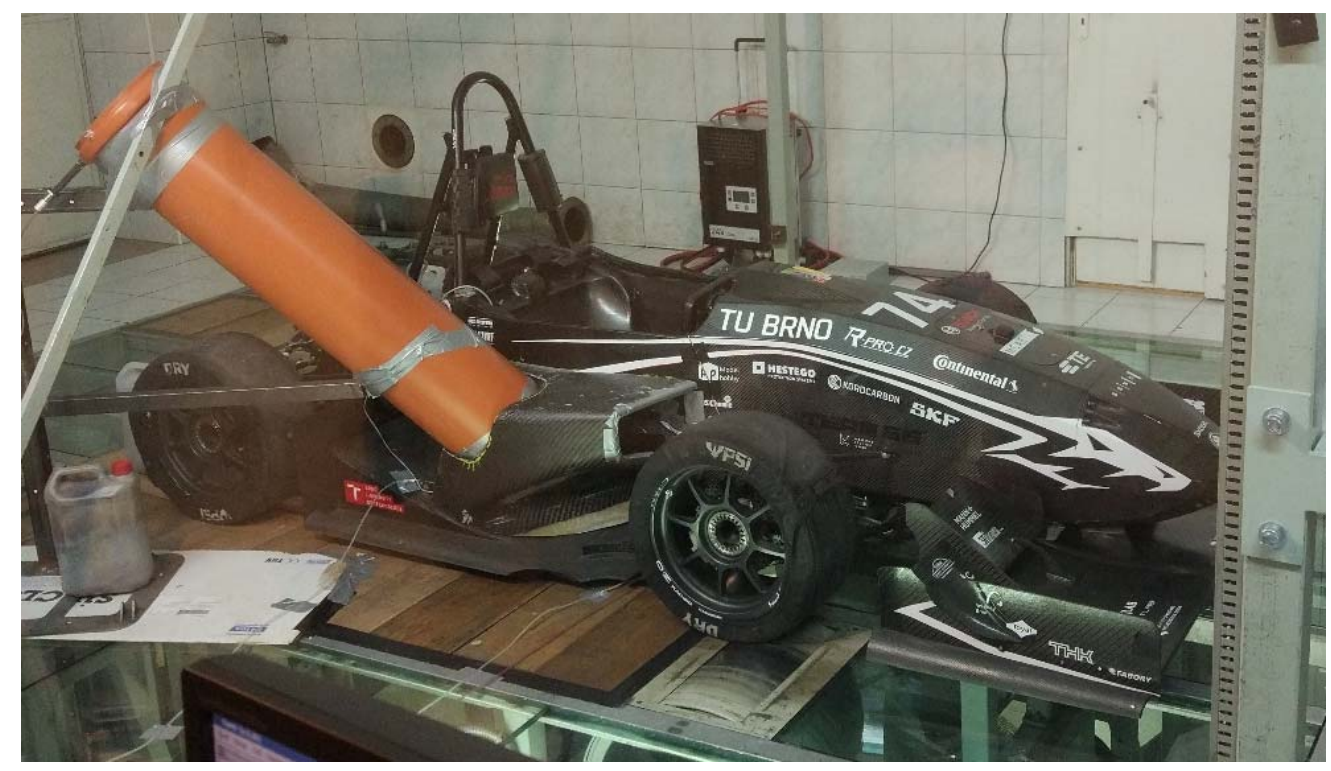

Fig. 1: Dragon 8 in wind tunnel.

\subsection{Measuring methods}

For measuring the air flow is used measuring propeller integrated in flow straighteners. Measurement is designed to measure fast and accurate airflow thru heat exchanger. Other possibilities are measuring pressure field at inlet of sidepod or use fan to measure airflow. Pressure field is less disturbing to car aerodynamics, but can't evaluate recirculation. Is possible only in one plane while inlet is angled and take significantly longer to measure one working point. Using fan without electrics motor as measuring device is possible only when there is possible to sacrifice fan mode during measurement.

Measuring section consist of round tube with flow straighteners and measuring propeller. Air is collected downstream of cooling fan and passes thru straight section to reduce vortexes. At the end of tube is positioned measuring propeller with revolution sensor to measure RPM of propeller. Measuring section is calibrated at known air flow and transfer function between propeller RPM and airflow is created.

Due to length of measuring section there isn't rear wing and sidepod is cut to connect tube directly to fan outlet. These modification influence air flow inside and at outlet of sidepod. Measured values will not be absolute correct, but for purpose of comparing different variants it provides good results.

During test there were measured also statics pressures inside sidepod at inlet side, pressure at outlet of heat exchanger before fan inlet and pressure at outlet of fan. From these measured pressures is possible to calculate pressure drop thru heat exchanger and therefore compare pressure drop with air mass flow. Pressure at inlet also indicate usage of ram air entering from inlet section of sidepod.

\subsection{Measured cases}

During measuring were measured five different variants of cooling packages. Base case is configuration with SPAL fan and unsealed space around heat exchanger inside sidepod. Second case consist of the same 
fan configuration and adding air guide from sidepod inlet to inlet of heat exchanger. Air is guided from whole inlet port and slowed down before entering heat exchanger. This air guide also seal space at HX inlet and eliminate recirculation. Third case is the same fan and space around HX is sealed with foam to eliminate recirculation. Fourth case is like base, but with fan switch OFF. Last case is equipped with two BLDC motors with smaller propeller and different fan shroud to accommodate two fans instead of one.
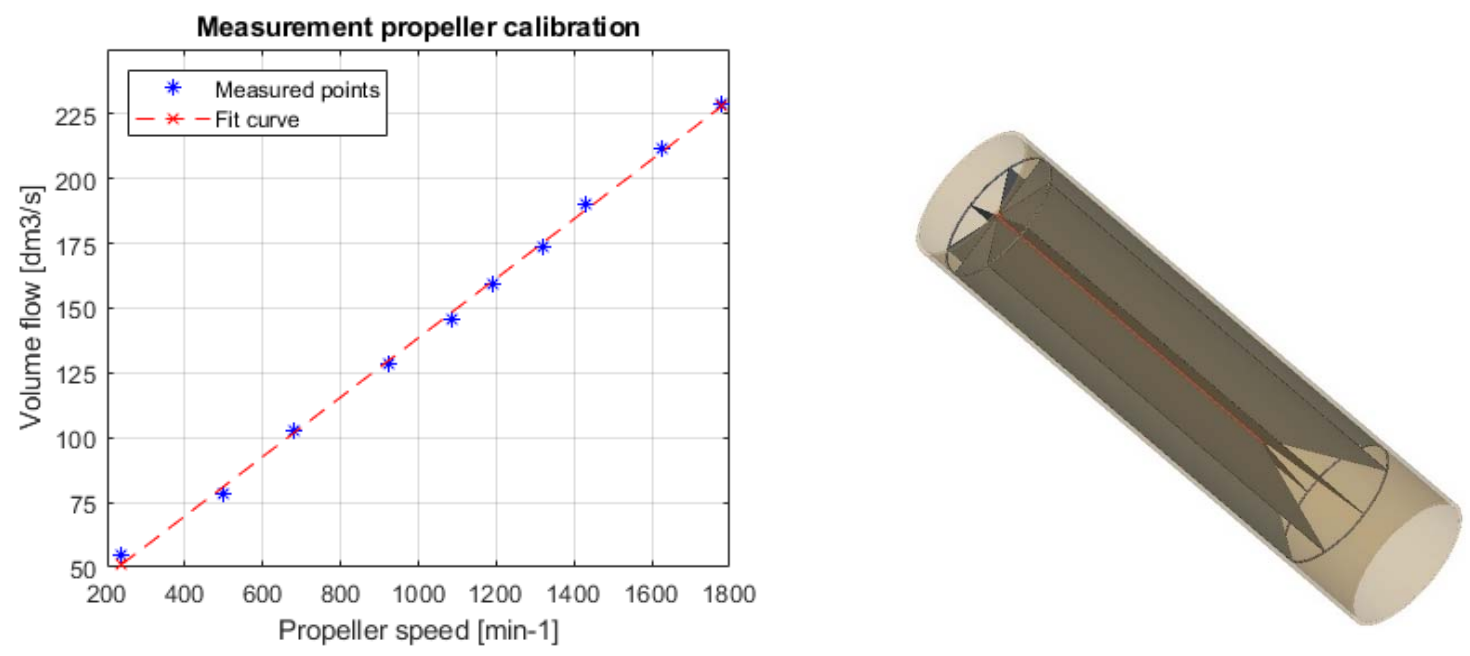

Fig. 2: Calibration and CAD data of measurement section.

\section{Result discussion}

From measured parameters most interesting is mass flow thru HX. From Fig. 3. we can observe that variants with SPAL fan have similar mass flow. There is difference in low speed for base variant in increased mass flow. This can be interpreted as desired outcome, but this increase is caused by recirculation air which come from back side of HX and not from sidepod inlet. Thus, air will be warmed by HX and decrease cooling capacity. This has been occurred at driving with car at low speed when inlet air had much higher temperature than ambient air. Second and third variants are similar in lower mass flow at low speed in comparison with base variant. This is caused by eliminating recirculation and allowing air to pass only thru sidepod inlet. However, at higher speed air guide show increase in mass flow utilizing better ram pressure from sidepod inlet. Variant with fan OFF show huge impact at mass flow without active sucking air and even at $100 \mathrm{~km} / \mathrm{h}$ do not provide same mass flow as fan at zero speed. BLDC motors are promising regarding weight saving and better control over motor speed, but propeller used at sucking air from HX is poorly designed for this task, thus lower mass flow can be observed. However, BLDC motors are more powerful than SPAL, they were regulated at same power consumption to be comparable with SPAL fan.

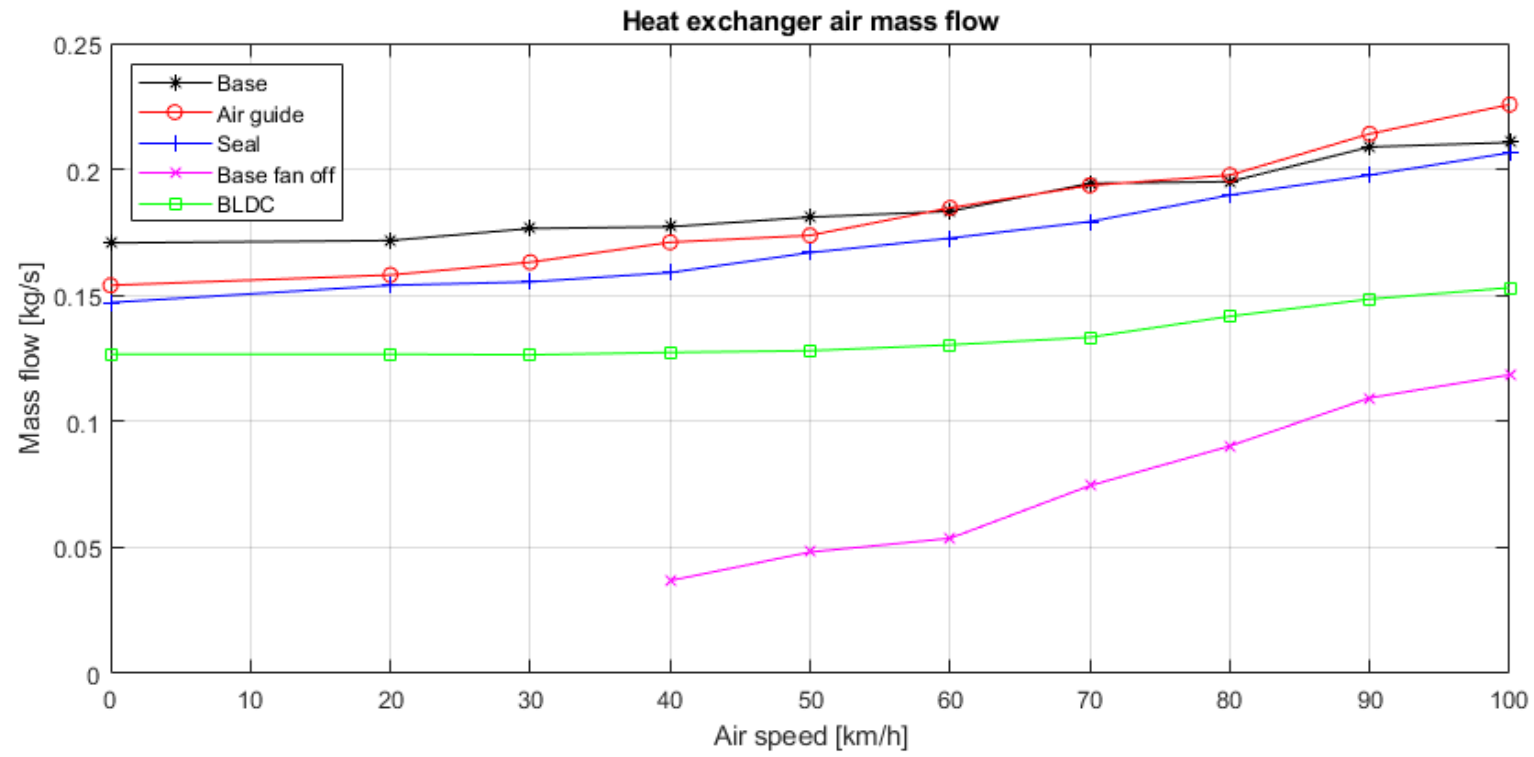

Fig. 3: HX mass flow. 
From measured pressures there is noticeable influence of increasing speed at inlet pressure. There is decrease in pressure at all cases except air guide. Air guide best utilized ram pressure and air is guided at inlet HX while speed is decreased thus pressure is increased. Sealed case shows lowest inlet pressure by offset caused by choking air at sidepod inlet and sucking by fan from zero speed. Pressure drop is computed from inlet and outlet pressure. There is apparent low mass flow for fan OFF and BLDC cases by lowest pressure drop confirming measuring mass flow. Base and seal cases have similar pressure drop for whole speed range. Best results show air guide by increased pressure drop at higher speeds and clearly highest mass flow from measured cases.
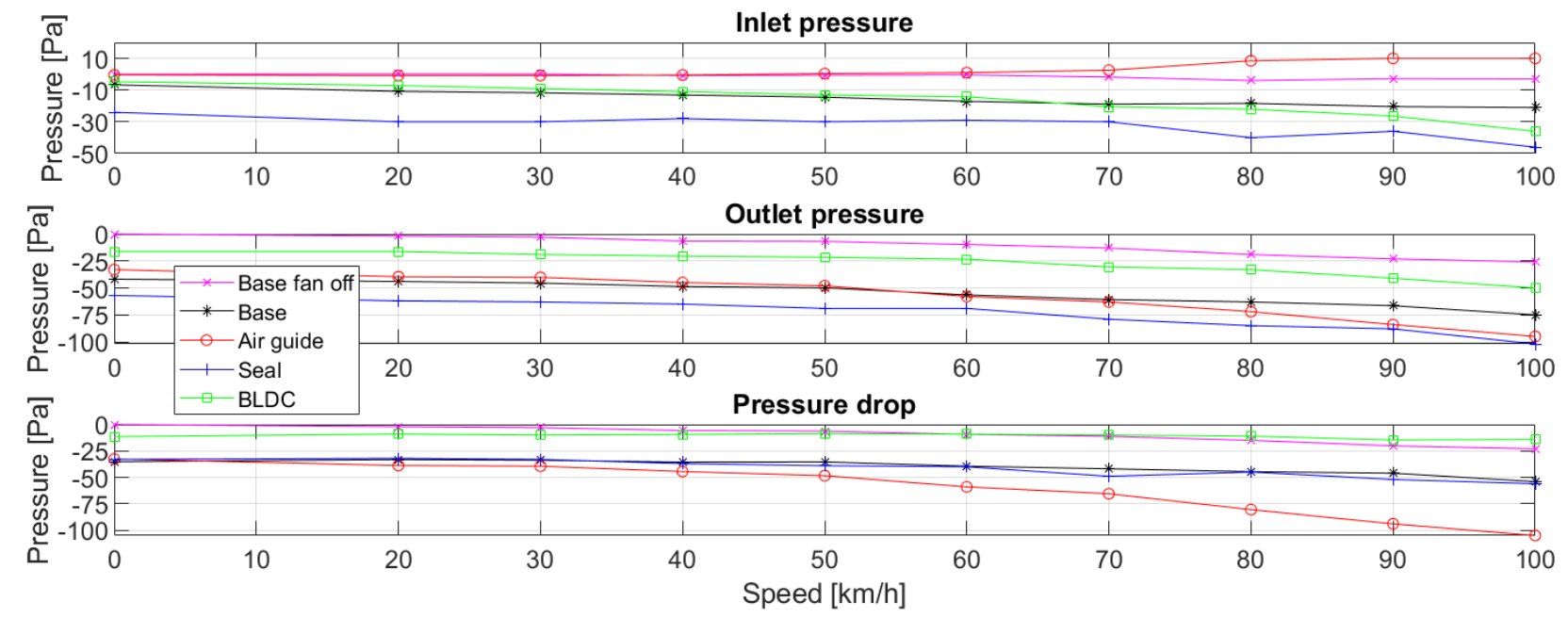

Fig. 4: Measured pressures.

\section{Conclusion}

Presented method for measuring air mass flow by propeller is capable of fast and reliable evaluating changes at cooling packages. There is disturbance at flow downstream of fan and space needed for installation is larger than other technique. Advantage of this method is short time to measure one working point but provide only integral value instead flow distribution. By simply measuring mass flow can be made wrong decision about recirculation at HX side. Therefore, pressure measurement is needed for evaluating this effects and proper post-processing is required for measured data. From measured cases best option is case with air guide. The is eliminated recirculation at low speed and at higher speed more air is available to engine cooling.

\section{Acknowledgement}

This work was realized using support of:

- Science fund at Brno University of Technology for year 2020.

- Jaroš company.

This support is gratefully acknowledged.

\section{References}

Aslam Bhutta, M. M., Hayat, N., Bashir, M. H., Khan, A. R., Ahmad, K. N., Khan, S. (2012). CFD applications in various heat exchangers design: A review. Applied Thermal Engineering. Elsevier Ltd.

Baskar S, Rajaraman R. (2015). International Journal of Thermal Technologies Airflow Management in Automotive Engine Cooling System-Overview. 1| International Journal of Thermal Technologies.

Shayler, P. J., Leong, D. K. W., Murphy, M. (2005). Contributions to engine friction during cold, low speed running and the dependence on oil viscosity. SAE Technical Papers. SAE International.

Singh, V., Tunestal, P., Tuner, M. (2017). A Study on the Effect of Elevated Coolant Temperatures on HD Engines. In SAE Technical Papers (vol. 2017-October). SAE International.

Šebela, K. (2019) Formula Student cooling system. Master thesis, BUT, Brno

Zhang, C., Uddin, M., Robinson, A. C., Foster, L. (2018). Full vehicle CFD investigations on the influence of frontend configuration on radiator performance and cooling drag. Applied Thermal Engineering. 